\title{
Evaluation of Power Grid Data Quality Based on State Estimation Results
}

\author{
Fujun Bao ${ }^{1, a^{*}}$, Miao Wang ${ }^{1, b}$, Qiang $\mathrm{Li}^{1, \mathrm{c}}$, Lei Wang ${ }^{1}$, Xiaochen $\mathrm{Ma}^{1}$ \\ ${ }^{1}$ China Electric Power Research Institute, China \\ abaofujun1991@126.com, bwangmiao@epri.sgcc.com.cn, Cliq@epri.sgcc.com.cn
}

\begin{abstract}
Keywords: Power grid data quality; State estimation; Evaluation index; Analytic hierarchy process. Abstract. The power grid data quality is the key factor which will affect various applications of smart grid power dispatch control system, and Power system state estimation results implied the problems in power grid data. The existing state estimation evaluation index is relatively single, and the analytic result of the power grid is not comprehensive. According to the indexes that affect the accuracy of state estimation, this paper puts forward a evaluation method of power grid data quality. The selection of evaluation indexes must follow with the identification and redundancy principles. Selected several typical indexes as an example, and used analytic hierarchy process to determine the weight of those indexes. Illustrated by the case of actual power system, the results show that the evaluation method is very effective and can reflect the hidden problem of grid model and data. Also, the evaluation result can provide guidance for the improvement of quality level of power grid model and data.
\end{abstract}

\section{Introduction}

The quality of basic model data is the key factor which affects various applications of smart grid power dispatch control system. Power system state estimation is the basic module of Energy Management System. Compare with the measured data, the state estimation can provide a more accurate, more logical and more complete data to the other power network analysis software [1].Therefore, the accuracy of state estimation result has a great impact on other applications.

In the domestic and abroad, some researches have been done to improve the accuracy of state estimation, such as the detection and identification of bad data, the robust state estimation, parameter identification and estimation, PMU mixed state estimation and so on[2-8]. But in fact, because we don't know the true value of the measurement, how to evaluate the state estimation result is a problem to be solved.

At present, it is not a lot of studies about the evaluation of the state estimation results, paper[9-10] put forward a evaluation index of estimation results under unknown state; based on cross entropy and zero injection mismatch, a new method for estimating the accuracy of state estimation is presented in paper[11]; the paper[12] puts forward some evaluation function of the given systems, such as linear function, quadratic function, square sum function, square sum exponential function, and so on; in paper[8], the diagonal elements of covariance matrix of the state estimation error is used to estimate the accuracy of the state estimation error; in paper[13], the estimation error variance and the variance of measurement error are compared. When the error variance of the estimation error is smaller than the original measurement error variance, the state estimation plays a filtering effect.

It has many factors which affect the accuracy of the state estimation results, such as the model of power network (topological relations, equipment parameters, model integrity), measurement data (measured bad data, measurement accuracy, impact load, measured time is not synchronized), etc. Despite the above research is done in some certain aspect of the state estimation, there are still lack of a comprehensive evaluation method of state estimation results.

In this paper, we analyze the current situation of the evaluation of the state estimation results, and put forward a comprehensive evaluation method based on some influence facts. Through the case of the actual power grid system, four typical indexes are chosen as an example to illustrate that the comprehensive evaluation method can reflect the problem in the data of network model, and to guide the improvement of the data integrity. 


\section{Present Situation of State Estimation Evaluation}

The State Grid Corporation provides two indicators for assessing the state estimation: the qualified rate of telemetry and the average of voltage residuals[14,15]. In 2013, the State Grid Corporation makes the qualified rate of telemetry as an index for performance check, the index is mainly determined by the residuals of estimated value and the measured value, measurement reference value and qualified threshold (active power $\leq 2 \%$, reactive power $\leq 3 \%$, voltage $\leq 0.5 \%$ ). This evaluation criterion which has a good guidance function to improve the quality of basic data and basic model has certain rationality, and it can improve the operation and application level of dispatch and control system of smart grid.

Theoretically, the qualified rate and the accuracy of the state estimation are evaluated with the error of state estimation and true value, but we can't get the true value of measurement. In fact, in the case of no bad data and model errors, the obtained state estimate value is credible when the convergence is calculated by the correct algorithm [16].

Actually, there are many factors which affect the accuracy of the state estimation results[17], such as the model of power network (topological relations, equipment parameters, model integrity), measurement data (measured bad data, measurement accuracy, impact load, measured time is not synchronized), etc.

Therefore, the single index of evaluation can lead to a high level of qualified rate, but the results are seriously deviated from the real state of the system [11], can not fully meet the needs of the follow-up application of the state estimation results, and can not be used to refine the difference of data, and can not reflect the problems of power grid basic data. In a sense, the qualified rate of telemetry reflects the error correction function of state estimation for the power grid model and the grid data.

Through the study of various evaluation index of state estimation, this paper shows that the multiple index comprehensive evaluation method can reflect the problem of model data of the power grid. This paper provides theoretical support for improving the quality of power grid data and the reliability of the state estimation results.

\section{Comprehensive Evaluation Method}

The comprehensive evaluation method uses several factors affecting the accuracy of the state estimation results, and makes an overall evaluation of the accuracy of the calculation results[18]. Namely, this method classifies and sorts the accuracy of the calculation results of the state estimation according to the given target.

Selection of Index. Compared with the previous evaluation method which only considers the effect of qualified rate of telemetry, the comprehensive evaluation method has the advantage of considering the effect of multiple index on the accuracy of state estimation results, such as model integrity, accuracy of parameters, accuracy of telemetry and correctness of teleindication action signal. In this method, the evaluation target is divided into different subsystems, and then subdivided into more specific index. Each evaluation subsystem has a certain independence, which can reflect the characteristics of the system, but also they can be combined in a fully reflection of the system status.

In this method, the factors that affect the accuracy of the calculation results of state estimation are divided into the model type, data type, and management type and so on. The model type is subdivided into topological relations, model parameters and model integrity. The data type is subdivided into the teleindication signal, the bad data, the measurement accuracy and the un-synchronized time of data. Management type mainly refers to the subordinate relationship between the power dispatch.

Principles of Comprehensive Evaluation Method. The optimization of the evaluation index is to make clear the independence and correlation between each index, and complete the logical test of single evaluation index and the integrated test of the comprehensive evaluation index.

For the comprehensive evaluation of the state estimation results, the following two basic principles are very important: the identification principle and the redundancy principle. "Identification" is the 
ability of the evaluation index to distinguish the characteristics of each evaluation target. In order to distinguish the level of basic data and basic model in different regions, such as the threshold of qualified rate will affect the qualified rate of telemetry, it is easy to appear many areas with the same qualified rate, the distinction is not obvious, while the accuracy of telemetry and regional objective function have a better distinguish ability than the qualified rate of telemetry.

"Redundancy" means that the degree of mutual independence between each index, which can be calculated by the correlation coefficient or similar coefficient. The redundancy is better, the more independent the index is. So those indexes can reflect the problem of the basic data and basic model from more angles.

\section{Typical Evaluation Index of State Estimation}

Qualified Rate of Telemetry in State Estimation Currently, the qualified rate of telemetry in state estimation is the most widely used evaluation index. It can be calculated by the formula:

$$
R_{\text {telemetry }}=\frac{N_{a}}{N} \times 100 \%
$$

The $R_{\text {telemetry }}$ is the qualified rate of telemetry in state estimation; $N$ is the total number of telemetry, include telemetry of bad data; $N_{a}$ is the number of qualified telemetry points which their telemetry estimated value is less than the threshold value.

Inaccuracy of State Estimation Telemetry. The inaccuracy of state estimation telemetry is the degree of deviation between the state estimation and the measured value. The state estimation results will be more accurate, if the inaccuracy of the state estimation telemetry get lower.

$$
J_{a}=\frac{1}{N} \sum_{i=1}^{N}\left(\frac{x_{i}-y_{i}}{\sigma_{i}}\right)^{2}
$$

$J_{a}$ is the inaccuracy of state estimation; $x_{i}$ is an element of the $N$ dimensional vector of state estimation value; $y_{i}$ is an element of the $N$ dimensional vector of measured value; $\sigma_{i}$ is an element of the $N$ dimensional vector of standard deviation of measurement error.

Unbalanced Degree of Bus Power. The unbalanced degree of bus power is the difference between the input bus power and the output bus power. It can not only reflect the accuracy and reliability of the measurement and control device, but also an important index to evaluate the balance between power supply and power consumption.

According to Kirchhoff's current law, the state estimates strictly meet the bus power balance condition, so the bus unbalanced power has a great impact on the state estimation results. The calculation formula is as follows:

$$
D=\frac{1}{m} \sum_{j=1}^{m} \frac{\left|S_{\text {in }}^{j}-S_{\text {out }}^{j}\right|}{S_{B}^{j}}
$$

$S_{i n}^{j}$ for the input power of the bus $j ; S_{\text {out }}^{j}$ for the output power of the bus $j ; S_{B}^{j}$ for the reference power of the bus $j$, which determined by the voltage level of bus $j ; m$ is the amount of the calculation buses.

Correct Rate of Teleindication Action Signal. In the process of power grid operation, there may be a wrong teleindication signal, which will lead to the power grid topology error, and become the hidden danger trouble of the power grid operation. The correct rate of teleindication action signal is a reflection of the problem of telemechanic equipment, switch status, signal transmission channel, was defined as:

$$
R=\left(1-\frac{N_{r f}+N_{i n}}{N_{o p}}\right) \times 100 \%
$$


$N_{r f}$ is the number of switch refused action; $N_{i n}$ is the number of switch incorrect action; $N_{o p}$ is the total number of switch action, the sum of the number of correct action ,refused action and incorrect action.

The accuracy of the state estimation model is ensured by the correctness of the teleindication action signal.

\section{Weight of Index}

The effect of each index on the evaluation object is different; we use the Analytic Hierarchy Process to determine the weight coefficient of each evaluation index [19,20].

AHP (Analytic Hierarchy Process) is a systematic analysis method which combines qualitative analysis and quantitative analysis. By comparing the important degree of the evaluation index, the judgment matrix is formed, and the eigenvector corresponding to the maximum eigenvalue of the judgment matrix is obtained. Then the normalized eigenvector is the weight of each evaluation index. Finally, the consistency of the judgment matrix is checked. If the check pass, the weight of index is reasonable; but if the check does not pass, the judgment matrix need to be re-adjusted, and the weight of index need to be re-calculate.

The results of the comprehensive evaluation on the state estimate can be recorded as:

$$
y=\sum_{i=1}^{n} w_{i} y_{i}
$$

$w_{i}$ is the weight of the evaluation index according to the judgment matrix; $y_{i}$ is the result of each evaluation index; and $y$ is the comprehensive evaluation result.

In order to eliminate the influence caused by the difference of order of magnitude and the difference of the dimension of each index , the evaluation index is needed to do the dimensionless data processing.

$$
y_{i j}^{*}=\frac{y_{i j}}{\sqrt{\sum_{i=1}^{n} y_{i j}^{2}}}
$$

$i$ is the serial number of different regions in each index; $j$ is the serial number of different index; $y_{i j}{ }^{*}$ is the numerical value of the index after dimensionless data processing.

\section{Case Analysis of Actual Power Grid}

In order to show the application of the comprehensive evaluation method in the actual power system, and demonstrate the rationality of the evaluation index, we select a certain state estimation result of the smart grid dispatch control system for analysis. The analysis of this section is to find out the problems in the network model and data by using the comprehensive evaluation method, and the weights of the selected indexes are 0.2.

The inaccuracy of active and reactive power telemetry, the unbalanced degree of bus active power are minimum indexes. The smaller value of these minimum indexes, the better result of evaluation. Also the qualified rate of telemetry and the correct rate of teleindication action signal are maximum indexes. If the value of these maximum indexes is bigger, the evaluation results will be better. By the method of vector normalization, the value of comprehensive evaluation index of each region is obtained according to the weight of each index. 
Table 1 Statistical information for state estimation results of a power grid

\begin{tabular}{|l|l|l|l|l|l|l|}
\hline Region & $\begin{array}{l}\text { Qualified } \\
\text { Rate of } \\
\text { Telemetry in } \\
\text { State } \\
\text { Estimation }\end{array}$ & $\begin{array}{l}\text { Inaccuracy } \\
\text { of Active } \\
\text { Power } \\
\text { Telemetry }\end{array}$ & $\begin{array}{l}\text { Inaccuracy } \\
\text { of Reactive } \\
\text { Power } \\
\text { Telemetry }\end{array}$ & $\begin{array}{l}\text { Unbalanced } \\
\text { Degree of } \\
\text { Bus Active } \\
\text { Power }\end{array}$ & $\begin{array}{l}\text { Correct } \\
\text { Rate of } \\
\text { Teleindicati } \\
\text { on Action } \\
\text { Signal }\end{array}$ & $\begin{array}{l}\text { Result of } \\
\text { Evaluation }\end{array}$ \\
\hline A & 99.88 & 0.0462 & 0.0034 & 14.543 & 100 & 0.345 \\
\hline B & 99.92 & 0.0088 & 0.0042 & 5.211 & 100 & 0.413 \\
\hline C & 99.97 & 0.0493 & 0.0022 & 3.178 & 100 & 0.429 \\
\hline D & 99.93 & 0.0033 & 0.0150 & 1.565 & 100 & 0.576 \\
\hline
\end{tabular}

Through the statistical information of Table 1, it can be seen that the Region $\mathrm{C}$ gets the highest qualified rate of telemetry in state estimation, but the Region $\mathrm{C}$ also gets the highest inaccuracy of active power telemetry. This shows that the Region $\mathrm{C}$ has a highly qualified rate of telemetry, but the estimated result of active power is the worst.

The statistical result of inaccuracy of active power telemetry in Region $\mathrm{C}$ is shown in Figure 1.

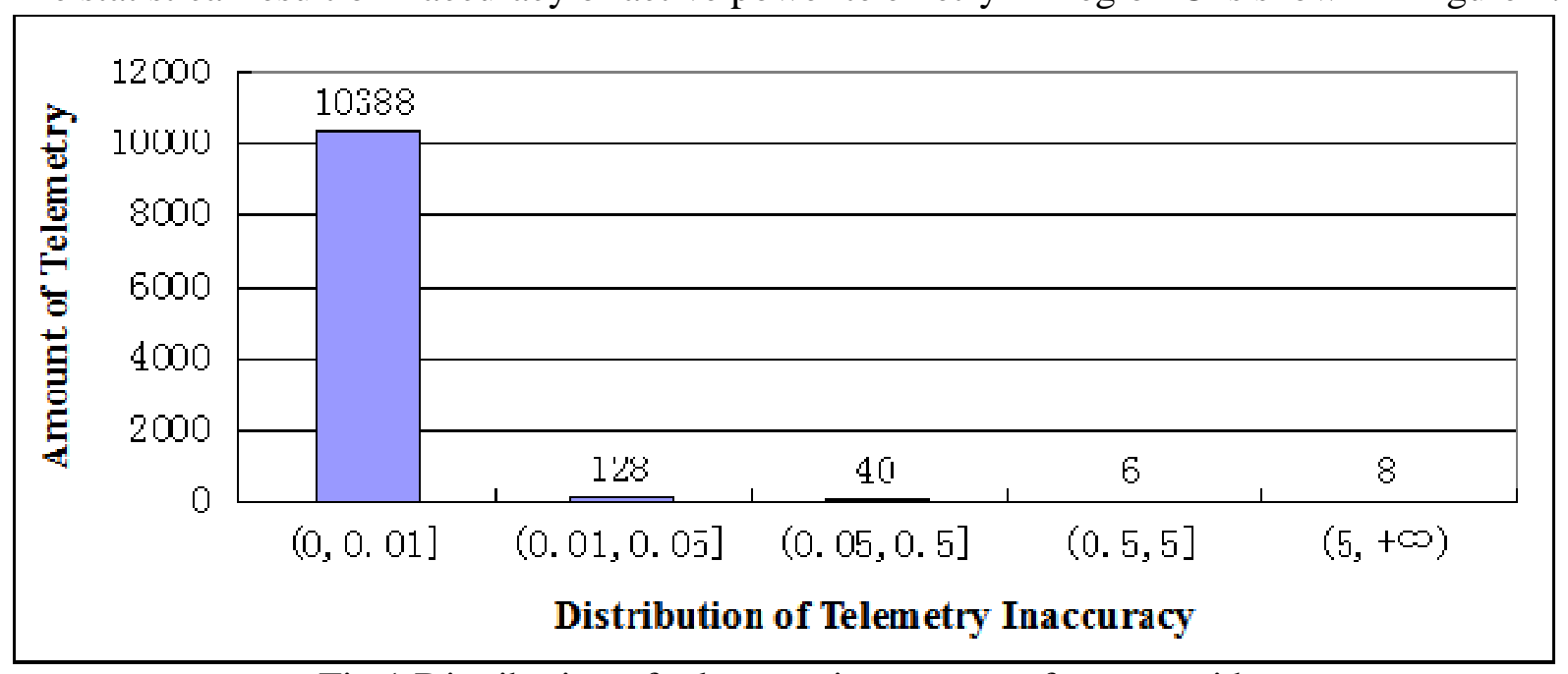

Fig 1 Distribution of telemetry inaccuracy of power grid

As we can see from Figure 1, most of the inaccuracy of telemetry is less than 0.01 . However, after analyze all the of telemetry, we found one inaccuracy is as high as 470.85 , which located in the bus with $86 \mathrm{MW}$ of unbalanced active power. After the problem of unbalanced active power has been solved, the change of the qualified rate of telemetry is small, but the inaccuracy of active power telemetry is reduced to 0.005 , and the unbalanced degree of bus active power is reduced to 2.86 .

The Region A has the lowest qualified rate of telemetry in state estimation, the inaccuracy of active and reactive power is not the highest, but its unbalanced degree of bus active power is much larger than that of other region. Therefore, we thought the mainly problem of Region A is the unbalance of bus active power. After the several main bus power are adjusted to balance which in Region A, the inaccuracy of active power telemetry is reduced to 0.016 , the inaccuracy of reactive power telemetry is reduced to 0.003 , and the unbalanced degree of bus active power is reduced to 10.583. Even so, there is still active power unbalance in some bus.

In the Region D, the accuracy degree of active power telemetry and the unbalanced degree of the bus active power are better than those of the Region C, but the qualified rate of telemetry is still lower than that of the Region C. It shows that the estimation of reactive power has a great influence on the qualified rate of telemetry in Region $\mathrm{D}$, and the problem is mainly concentrated on the telemetry of reactive power and voltage. At the same time, based on the value of comprehensive evaluation index, the Region D is better on the quality level of data than Region C, but Region D is worse on qualified rate of telemetry than Region $\mathrm{C}$. It shows that the inaccuracy of active power telemetry and unbalanced degree of bus active power have a good effect on distinguishing the quality level of power grid basic data. 
In summary, the single qualified rate of state estimation can not fully reflect the level of the state estimation and the hidden problems, combined with the accuracy degree of the telemetry, the unbalanced degree of the bus power and the accuracy degree of the teleindication action signal, can provide a higher distinguish degree of the evaluation of the state estimation results, but also can reflect the hidden problem of the grid model and data.

\section{Summary}

The existing state estimation evaluation index is single, and the analytic result of the power grid is not comprehensive. Based on the indexes that affect the accuracy of state estimation result, this paper puts forward a comprehensive evaluation method of state estimation. There are two basic principles of index selection are very important: the principle of identification and the principle of redundancy. By selected the qualified rate of telemetry, the inaccuracy of telemetry, the unbalanced degree of bus power and the correct rate of teleindication action signal as four evaluation indexes, and illustrated by the case of actual power system, the results show that the evaluation method is very effective and can reflect the hidden problem of grid model and data. Also, the evaluation result of state estimation can promote the quality level of basic data of the power grid.

\section{Acknowledgment}

This work is supported by State Grid Corporation of China(DZ71-15-046) and State Grid Fujian Electric Power Company Limited.

\section{References}

[1] BYUN J, HONG I, KANG B, et al. A smart energy distribution and management system for renewable energy distribution and context-aware services based on user patterns and load forecasting[J].IEEE Trans on Consumer Electronics, 2011, 57(2). 436-444.

[2] YAN Quanchun, WEI Zhinong, SUN Guoqiang, et al. A robust WLAV state estimation based on multiple predictor-corrector interior point method[J]. Power System Technology, 2013, 37(8). 2195-2197 (in Chinese).

[3] LU Zhigang, WANG Haorui, SUN Jika. Optimal data screening and bad data identification based on sensitive analysis[J]. Power System Technology, 2011, 35(2) 39-40(in Chinese).

[4] NING Liaoyi, SUN Hongbin, WU Wenchuan, et al. State estimation based branch parameter estimation method for power grid [J]. Proceeding of the CSEE, 2009, 29(1). 8-10(in Chinese).

[5] G.P. Granelli, M. Montagnaa, Identification of interacting bad data in the framework of the weighted least square method [J]. Electric Power Systems Research, 2008, 78(5). 806-814.

[6] WANG Keying, MU Gang, CHEN Xueyun. Precision improvement and PMU placement studies on state estimation of a hybrid measurement system with PMUS[J]. Proceeding of the CSEE, 2001, 21(8). 30-32(in Chinese).

[7] Mili L, Cheniae M.G, Rousseeuw P.J., Robust state estimation of electric power systems[J], IEEE Trans. on Circuits and Systems-I: Fundamental theory and applications, 1994, 41(5).

[8] Liu W H E, Wu F F, Liu S M. Estimations of parameter errors form measurement residuals in state estimation[J]. IEEE Trans. on Power Systems, 1992, 7(1). 81-89.

[9] HE Guangyu, DONG Shufeng. Power system static state estimation based on uncertainty of measurement Part One Result Evaluation [J]. Automation of Electric Power Systems, 2009, 33(9). 21-22(in Chinese). 
[10] CHANG Naichao, Wang Bin, He Guangyu, Xin Yaozhong, Zhang Zhigang. An Improved Algorithm for State Estimation Based on Maximum Normal Measurement Rate[J]. Automation of Electric Power Systems, 2014,38(11). 62-67

[11] GUO Ye, ZHANG Boming, WU Wenchuan, et al. Research on the appraisement of state estimation in practical power systems [C]. Proceeding of 2012 Automation of Electric Power Systems Special Committee Academic Communications and Discussion Conference, 2012. 2-4 (in Chinese).

[12] Takata H., Uchino E., Takata S., A judging function of the state-estimation accuracy and its application to the electric power system[J], IEEE Trans. on Power Apparatus and Systems, 1981, 100(12). 5049.

[13] LI Dalu, LI Rui, SUN Yuanzhang. Data compatibility analysis of WAMS/SCADA hybrid measurements state estimation[J]. Proceeding of the CSEE, 2010, 30(16). 61(in Chinese).

[14] State Grid, Practical acceptance method of dispatch and control system for smart grid [S]. 2013, 7. 25-28.

[15] State Grid, Practical requirement of dispatch and control system for smart grid [S]. 2013, 7. 7 .

[16] Gu JINwen. Disagreements discussion on state estimation in related standards[J]. Automation of Electric Power Systems, 2014, 38(1). 134.

[17 ]Huangtao, Lu Jiangang, Zhanghui. Adjustable measures to improve calculation precision of state estimation in Guangdong province[J]. Power System Technology, 2004, 28(16). 78-81.

[18] SU Weihua. Issues study on theory and method of multiple objective comprehensive evaluation[D]. Xiamen. Xiamen University, 2000. 28.

[19] LI Juan, XUE Yong-rui, XU Bing-yin, et al. An Analytic Hierarchy Process Based Assessment Method for Power System Transient Models[J]. Power System Technology, 2013, 37(8).2209.

[20] CAO Guo-qing, XING Jin-cheng, TU Guang-bei. Grey Method with Use of an Analytic Hierarchy Process for Performance Evaluation of Flue Gas Desulfurization Technology[J]. Proceeding of the CSEE, 2006, 26(4).53. 